\title{
Erratum: Robust calibration of a universal single-qubit gate set via robust phase estimation [Phys. Rev. A 92, 062315 (2015)]
}

\author{
Shelby Kimmelø, Guang Hao Low, and Theodore J. Yoder
}

(Received 22 October 2021; published 1 December 2021)

DOI: 10.1103/PhysRevA.104.069901

In Eq. 5.5 in Sec. V, we state that the probability of an error occurring at the $j$ th iteration is given by the probability that the estimate is outside of $\pi /\left(2 k_{j}\right)$ of the actual value. However, as pointed out in Refs. [1,3], an error can, in fact, occur even when the estimate at the $j$ th round is within $\pi /\left(2 k_{j}\right)$ of the true value. The correct condition that characterizes when an error occurs is that the estimate is outside of $\pi /\left(3 k_{j}\right)$ of the actual value [1,3]. Thus, whereas the detailed analysis of Appendix $\mathrm{C}$ does bound the probability that the $j$ th iteration estimate is within $\pi /\left(2 k_{j}\right)$ of the true value, this only gives a lower bound on the probability of an error occurring.

In fact, Ref. [2] already bounds the probability that the estimate at the $j$ th round is within $\pi /\left(3 k_{j}\right)$ of the actual value and Ref. [1] provides a tighter analysis - so whereas our analysis in Sec. V A is not correct, Refs. [1,2] show that the general procedure still attains Heisenberg scaling with a slightly worse constant overhead than the performance claimed in the original paper. A simple argument in Appendix B of Ref. [3] then shows how to leverage those analyses to obtain (through an additional constant overhead) a procedure with Heisenberg scaling that is robust to additive errors, superceding Sec. V B and showing the main result of the original paper-that phase estimation can provide robust calibration of a universal single gate set-is still valid.

[1] F. Belliardo and V. Giovannetti, Achieving heisenberg scaling with maximally entangled states: An analytic upper bound for the attainable root-mean-square error, Phys. Rev. A 102, 042613 (2020).

[2] B. L. Higgins, D. W. Berry, S. D. Bartlett, M. W. Mitchell, H. M. Wiseman, and G. J. Pryde, Demonstrat- ing heisenberg-limited unambiguous phase estimation without adaptive measurements, New J. Phys. 11, 073023 (2009).

[3] A. E. Russo, W. M. Kirby, K. M. Rudinger, A. D. Baczewski, and S. Kimmel, Consistency testing for robust phase estimation, Phys. Rev. A 103, 042609 (2021). 